\title{
In Vitro Propagation and Cellular Behaviour Studies of Severinia buxifolia (Poir.) Tenore
}

\author{
H. Elias, R.M. Taha, N.A. Hasbullah, N. Mohamed and S. Abdullah \\ Institute of Biological Sciences \\ Faculty of Science \\ University of Malaya \\ 50603 Kuala Lumpur \\ Malaysia
}

Keywords: chromosome counts, mean cell and nuclear areas, mitotic index, MS media, nuclear DNA content, ploidy level, plant regeneration

\begin{abstract}
In this study, a wild relative of Citrus, Severinia buxifolia, was subjected to tissue culture treatments in order to identify the best medium for plant regeneration of this species. This research was carried out due to its range of uses and because of its potential to be a rootstock species that is tolerant to boron. Different sources of explants were cultured on Murashige and Skoog (MS) media supplemented with various types and concentrations of hormones. Results indicated that responsive explants were from stems and cotyledons, based on the formation of shoots. The best shoot formation was observed on MS medium supplemented with $0.5 \mathrm{mg} / \mathrm{L} \mathrm{NAA}$ and $1.0 \mathrm{mg} / \mathrm{L}$ BAP. The studies also included cellular parameters such as Mitotic Index (MI), chromosome counts, ploidy level determinationand nuclear DNA content, and mean cell and nuclear areas. Investigation of the changes that occurred as the plants were transferred from in vivo to in vitro conditions were also made. The results showed that the chromosome number in vitro was higher than in vivo. Besides, changes in MI, ploidy level or nuclear DNA content, mean cell and nuclear areas were detected which could lead to somaclonal variations.
\end{abstract}

\section{INTRODUCTION}

In vitro propagation techniques have been extensively developed for a large number of plant species including tropical and subtropical fruits. The technique has become an effective component in plant breeding where it is used for methods including mass propagation and preservation of elite plants. Through tissue culture, the plantlets grown are true-to-type, which is one of the most important characteristics for researchers to optimize effectiveness of their research. Severinia buxifolia (Poir.) Tenore is one of the wild relatives of citrus, classified in the Citrinae subtribe. It is commonly known as 'boxorange' or 'severinias' and can be found growing in South China, Peninsular Malaysia, East India, The Philippines, Kei Island and New Guinea. Jones et al. (1987) reported that the two wild Severinia species found growing in Malaysia were Severinia buxifolia and Severinia disticha. The first taxonomic studies of Severinia buxifolia were reported by Poiret (1797), followed by Bentham (1851) and then Swingle (1967). The species has oval green leaves with thorns and oblong black berry fruits. Studies by Tinggie (1990) showed that the species was monoembryonic where one seed produces one seedling. The leaves are used for production of yeast, normally called 'tsau ping lak' in China, to bake cakes. Furthermore, the species has shown immunity toward diseases and tolerance to boron and high concentrations of salt. Based on these advantages, the species has ideal characteristics for application as a 'rootstock' (Swingle, 1967).

The main aim of this research was to establish a protocol for in vitro propagation of Severinia buxifolia due to very limited previous research on the species. The optimum media for regeneration was observed and the effects of light, sucrose concentrations, hormones, $\mathrm{pH}$ and explant sources were evaluated. The studies also involved investigating cellular behaviour such as mitotic index, chromosomes counts, ploidy level or nuclear DNA content, and mean cell and nuclear areas. 